\title{
Exploring New PLTL Modalities, Forging New Alliances
}

Nadia Stoyanova Kennedy

New York City College of Technology

nkennedy@,citytech.cuny.edu

Ariane M. Masuda

The City University of New York

\section{Recommended Citation}

Kennedy, N. S., \& Masuda, A. M. (2021). Exploring New PLTL Modalities, Forging New Alliances. Advances in Peer-Led Learning, 1, 44-54. Online at https://doi.org/10.54935/apll2021-01-05-44 


\title{
Exploring New PLTL Modalities, Forging New Alliances
}

\author{
Nadia Stoyanova Kennedy* and Ariane M. Masuda \\ Department of Mathematics \\ New York City College of Technology, The City University of New York \\ Brooklyn, New York 11201 \\ *nkennedy@,citytech.cuny.edu
}

\begin{abstract}
This essay focuses on rethinking and reimagining elements of a PLTL program, and on the new modalities to meet challenges of online undergraduate mathematics courses and rising demands for flexible student support. It examines advantages and challenges as found in the Integrated PLTL and Virtual Peer-Led Mathematics Study Groups, including issues such as meeting protocols, and the selection and training of peer leaders. Finally, it discusses an alliance with the college's mathematics education program, which allows the PLTL program to draw on senior prospective teachers to co-organize and facilitate virtual study groups supporting undergraduate mathematics courses.
\end{abstract}

Keywords: Higher Education, Peer-Led Team Learning, Peer Leader training, Undergraduate Mathematics Foundational Courses, Academic Support, Mathematics Education Students 


\section{Introduction}

Peer-Led Team Learning (PLTL) has proven to be an effective and high-impact practice for many institutions (Keup, 2016). It has been widely implemented in foundational undergraduate courses to decrease the historically high drop-fail-withdraw rates (Liou-Mark et al., 2013; Kokkelenberg, Dillon \& Christy, 2008; Lee \& Choi, 2010). Results have shown that the mathematics courses with PLTL workshops have at least $15 \%$ higher pass rates and at least 15\% lower withdrawal rates (Liou-Mark et al., 2013). Other studies of the effects of PLTL in any given course have shown that the PLTL student population has a significantly higher passing rate compared to the non PLTL one (Cracolice \& Deming, 2005). The PLTL program is shown to be highly effective for first-year underrepresented minority STEM students (Liou-Mark et al.; Liou-Mark et al., 2015; Liou-Mark et al., 2018). It has also been found that among the introductory biology students, those who participated in PLTL averaged more than a letter grade above those who did not (Snyder et al., 2015). PLTL programs also have benefits for Peer Leaders, who develop leadership skills and grow as group facilitators (Chase et al., 2020; Hoiland, Reyes \& Varelas, 2020).

For two decades, the PLTL instructional model has been successfully utilized at a nonresidential, undergraduate comprehensive college that is part of a large urban, public university in New York City. The college is designated as a Hispanic-Serving Institution (HSI) with more than 17,300 students, of whom 63\% are African American or Hispanic. It has a large portion of nontraditional students, about $40 \%$ of whom are part-time, and about $27 \%$ of whom report working 20 or more hours per week. Over 60\% are first-generation college students. PLTL has provided essential support to this population, in particular to first-year underrepresented minority STEM students and has consistently shown to increase course pass rates and lower withdrawal rates (Liou-Mark et al., 2013; Liou-Mark et al., 2015; Liou-Mark et al., 2018). The model has been successfully implemented in various STEM disciplines, most extensively in mathematics courses. Courses such as chemistry and statics have also employed PLTL in their lab components.

\section{The Evolution of the PLTL Program}

The PLTL program was initially adopted to support students, particularly those who are underrepresented minorities, first-generation students, and students with long interruptions after high school. Another program goal was to mitigate low retention rates among STEM-degree enrolled students. The approximate average retention rates are $70 \%$ for STEM baccalaureate degree-enrolled freshmen and 57\% for students enrolled in associatedegree STEM programs. The same rates for Black and Hispanic students are even lower- 
$66 \%$ and 52\%, respectively (the percentages given are the five-year averages for 2015, 2016, 2017, 2018, and 2019). The PLTL program was initiated in the early 2000s, and actively recruited undergraduates majoring in science, technology, engineering, and mathematics disciplines to train as Peer Leaders for STEM workshops.

Typically, Precalculus or higher-level mathematics instructors encourage their outstanding students to become Peer Leaders. The program has two main Peer Leader training components — a one credit Peer Leader training course, and ongoing mentoring. The Peer Leader Training in Mathematics course meets once a week for 50 minutes over the course of 15 weeks. The course is typically taught by a mathematics education professor. It is required for all novice Peer Leaders, who are simultaneously assigned to one of the courses that includes a PLTL workshop — that is, STEM foundation courses in chemistry, civil engineering, and mathematics. The Peer Leader Training in Mathematics course focuses on introducing students to learning and communication theory, group dynamics and facilitation of group problem solving, whether in a mathematics course or in a chemistry or static lab activity. After this initial semester of training, Peer Leaders remain mentees of experienced Peer Leaders and to participate in biweekly workshops.

The PLTL workshop model consisted of stand-alone one-hour workshops that met once a week.-Each such workshop was associated with a particular section. There were two to three Peer Leaders, at least two of whom had previous experience in the program. A novice Peer Leader assigned to such a workshop apprenticed with the more experienced ones and followed their guidance. Under this Peer Leadership, the group's 8-12 students worked collaboratively on a set of problems. Students were required to attend the mathematics and civil engineering workshops, and strongly encouraged to participate in the chemistry workshops. The Peer Leaders maintained attendance records and completed other tasks assigned by the course instructor. The workshops varied to some degree for each discipline, but for many years the independent workshop was the single modality employed.

From 2017 to 2020, the PLTL program and the Mathematics Department experimented with integration of the PLTL workshops into some of the College Algebra and Trigonometry and Quantitative Reasoning courses. Students with lower placements scores enrolled in the College Algebra and Trigonometry and Quantitative Reasoning sections with mandatory corequisite integrated PLTL workshops. Instead of a stand-alone one-hour workshop, which typically followed one of the weekly course sessions, the workshop was integrated into that meeting. An Integrated PLTL model involved one or two 20-30 minute "spells" of group work guided by one or two Peer Leaders. The Peer Leaders, who were present during the entire class, "stepped in" during the problem-solving periods of the session 
to support the group's work. This modality was found to meet the increased demand for more Peer Leaders. Course instructors decided the timing and duration of the group problem solving "spells," and other selected tasks. Thus, in the two years preceding the COVID-19 pandemic in 2020, there were two PLTL modalities operating--Integrated and Independent. Typically, between 30 and 40 sections of our undergraduate mathematics and statistics courses utilized PLTL each semester in one or another of these modalities, mostly in Remedial Mathematics, Quantitative Reasoning, College Algebra and Trigonometry, Precalculus, Calculus, and Statistics courses.

\section{$\underline{\text { Pandemic Disruptions }}$}

During the early stage of the COVID-19 pandemic in Spring 2020, we faced enormous academic challenges caused by the rapid transition to remote classes, most particularly in student engagement and support. As all mathematics courses transitioned to synchronous remote learning, the initial plan to transition any linked independent and integrated workshops did not seem feasible. Due to technological and other pandemic-related issues, we were only able to offer a limited number of the workshops that typically supported our courses.

Remedial Mathematics and Quantitative Reasoning courses, which used Integrated PLTL, were assigned one or two Peer Leaders. Each course instructor assigned them to work with groups during the problem-solving periods of the session in order to support the groups' work in breakout rooms on Zoom or Blackboard Collaborate. The Peer Leaders moved systematically through each virtual room. In the Spring and Fall 2020, this modality had various degrees of success. The transition from in-person to virtual synchronous environment was difficult. Some instructors simply lacked pedagogical and technological knowledge and skills that supported efficient implementation of the workshops in the virtual environment.

The biggest challenge was in implementing online workshops for numerous sections of the College Algebra and Trigonometry course, of which we had 13 faster and 30 slowerpaced sections. Each section met for one hour and forty minutes every session. However, the slower paced sections met three times a week, and faster-paced ones met twice a week. Each of the former were assigned one Peer Leader to work with the instructor, but the latter were assigned none. Typically, students in the course need support, and the demands of the virtual environment exacerbated this need. Given the rapid transition to remote learning, compounded by the dubious implementation of the integrated PLTL in some sections and the lack of Peer Leader support in others, we decided to implement an additional level of student support. This was the context in which a third modality of PLTL was conceived and adopted, 
Virtual Peer-Led Mathematics Study Groups. Designed with the purpose of supporting the large group of students enrolled in all College Algebra and Trigonometry courses.

\section{Virtual Peer-Led Mathematics Study Groups}

Two Virtual Peer-Led Mathematics Study Groups were formed, each of which convened once a week for 90 minutes. Each meeting focused on a selected weekly course topic that had been discussed in class. One of the study groups focused on topics of the faster-paced sections, and the other focused on topics of the slower-paced sections. Study group meeting hours and topics were announced in advance and widely advertised, and any College Algebra and Trigonometry student could join either of the groups. Four Peer Leaders were assigned to conduct the study groups, two of whom would work collaboratively in planning and facilitating each group.

Each group meeting on Zoom followed a specific protocol. One of the Peer Leaders began with a 15-minute review and modeling of a problem. The leader used questioning techniques utilized in mathematics teacher preparation to orchestrate productive mathematics discussions (e.g.,., Boaler, Humphreys, 2005; Chapin, O’Connor, \& Anderson, 2009). The aim was to engage the attending students and facilitate collectively solving the given problem, and to offer this collective problem-solving as a model for the small group deliberations. The modeling with the whole-group was followed by small-group problem-solving alternating with whole-group debriefs. The students were assigned two sets of problems and split into small groups to work on them in Zoom breakout rooms. The two Peer Leaders, moved through the breakout rooms to help with clarifications, offer suggestions and provide necessary scaffolding. After 20-30 minutes, all students were called back to the main room for a quick debriefing of the assigned problems. The Peer Leaders followed the cycle of smallgroup work and whole-group debrief with each of the two problem sets. In designing the meeting protocol shown in Table 1 below, we considered the best mathematics teaching and learning practices. For example, principles of "responsive pedagogy," whereby the group facilitator consciously attends to and responds to students' thinking, thereby encouraging student voice and agency (e.g., Lampert \& Graziani, 2009; Robertson et al., 2014) in the problem-solving process. Careful attention was paid to the coherence and sequencing of the selected math problems and their cognitive demand, in order that "the level of challenge should be conducive to what has been called 'productive struggle'" (Schoenfeld, 2014). 
Table 1. A Sample Meeting Protocol

\begin{tabular}{|l|l|}
\hline \multicolumn{2}{|l|}{ Virtual Peer-Led Mathematics Study Group Meeting Protocol Meeting takes place on Zoom } \\
\hline Timing & Activity \\
\hline $\begin{array}{l}\text { (Main room) } \\
\text { Brief Topic Review \& Modeling Problem Solving } \\
\text { Model Problem Solving. Choose an appropriate problem. } \\
\text { Solicit ideas from students; solve it collectively in whole-group discussions; Highlight } \\
\text { main ideas, generalizations, conclusions. }\end{array}$ \\
\hline $\begin{array}{l}\text { Small-Group Collaborative Problem Solving } \\
\text { (Breakout rooms) } \\
\text { Assign problem set \#1. } \\
\text { Split the participating students into small groups (4-6). } \\
\text { Assign to breakout rooms. }\end{array}$ \\
\hline $\begin{array}{l}\text { Whole-Group Debrief of Problem Set \#1 } \\
\text { Fain room) }\end{array}$ & $\begin{array}{l}\text { Feature groups' solutions, ask the group to explain, clarify and/or add information } \\
\text { if necessary. }\end{array}$ \\
\hline (Breakout rooms) & $\begin{array}{l}\text { Small-Group Collaborative Problem Solving } \\
\text { Split the participating students in small groups (4-6). } \\
\text { Assign to breakout rooms. }\end{array}$ \\
\hline $\begin{array}{l}\text { Whole-Group Debrief of Problem Set \#2 } \\
\text { (Main room) }\end{array}$ & $\begin{array}{l}\text { Feature groups' solutions, ask the group to explain, clarify and/or add information } \\
\text { if necessary. }\end{array}$ \\
\hline Total: 90 min & Meeting ends. \\
\hline
\end{tabular}

The elements of the meeting protocol require that Peer Leaders are well trained in group facilitation and mathematical pedagogical knowledge and skills. Under the protocol, leaders must be able to highlight important ideas, to clarify and dispel misconceptions, and to make suggestions for directions that the students' problem-solving process might take. They must be able to switch between facilitating whole-group discussions, small-group discussions, and debriefing. Since this level of facilitation knowledge and skills might exceed the training that Peer Leaders may have, we take care to recruit the four Peer Leaders from the seniors in the college's undergraduate mathematics education program. All of them have acted as Peer Leaders in previous semesters. In fact, all mathematics education students apprentice as Peer Leaders at some point in their undergraduate study, as the one-credit Peer Leader Training in Mathematics course is required. Most of the mathematics education students continue to be Peer Leaders in the semester following their initial training. Our observations show that they tend to make excellent Peer Leaders, as they are also trained in facilitating whole-class discussions and small-group work, and in engaging students in higher-order cognitive activities that involve fostering conceptual understanding through a pedagogy of questioning. The latter have been deemed as important factors for successful PLTL practice and supported by at least two research reviews (Hung et al., 2008; Strobel \& van Barneveld, 2009). Indeed, 
before the study groups commence, the two faculty and four designated Peer Leaders perform several training "rehearsals" - an enactment of activities, including transitions from wholegroup to small-group activities and other routines (e.g., opening breakout rooms, assigning students to small groups, etc.) (Lampert \& Graziani, 2009).

Since the Virtual Peer-Led Mathematics Study Groups include students from all College Algebra and Trigonometry course sections, they operate relatively independently of the course instructors. All organizational work is coordinated by two faculty members (the authors of this essay) who, along with the four mathematics education students chosen to be the Peer Leaders, design plans and protocols for the study group meetings, and samples of meeting activities. One useful curriculum resource is the recently designed departmental Course Hub (https://openlab.citytech.cuny.edu/mat1275coursehub/lessons/). All study group activity sheets, which are distributed during each session, are subsequently deposited into a DropBox folder and made available to students.

Adequate Peer Leader training is considered an essential component of any Peer Leader program (e.g., Becvar, Dreyfuss, Dickson, 2008; Hung et al., 2008). Peer Leaders need to be good at facilitating group interactions, diagnosing and managing misconceptions, helping student clarify ideas and effectively scaffolding student communication (e.g., Becvar, Dreyfuss, Dickson, 2008; Narode, 2012). It is difficult to measure the exact impact of Peer Leader training on student achievement as it is an indirect one, and is mediated by other factors, such as the difficulty level of the tasks students engage with, and other student performance variables. However, a few studies have shown that Peer Leaders who are trained and perform in accordance with the PLTL pedagogy of facilitation contribute to more sustained student communication and conceptual understanding. A study conducted by Brown, Sawyer, \&Frey (2009a) showed that Peer Leaders who predominantly employed facilitative interactional style had a higher percentage of group student-to-student interactions and fostered more equal student participation. Conversely, Peer Leaders who used an equal amount of instructional and facilitative discourse or a predominantly instructional one seemed to have contributed to unequal group participation and shorter group interactions. A subsequent study found that when Peer Leaders used facilitative discourse and provided ample scaffolding students not only engaged in extended discussions, but also exhibited the development of conceptual understanding (Brown, Sawyer, \&Frey, 2009b). These findings are consistent with research in mathematics education which points to teachers/instructors as among the most influential factors in improving student performance (e.g., Hill et al., 2005).

Studies also show that Peer Leaders often misconstrue their role in supporting students, or revert to lecture models that they have been exposed to as students (e.g.,. Clark 
and Raker, 2020). Such results raise concerns about inadequate or insufficient Peer Leader training and call for rethinking the ways in which Peer Leaders are selected and trained. Given that mathematics and science education students undergo extensive training in organizing and facilitating productive discussions, detecting student misconceptions and scaffolding student ideas, we consider alliances with mathematics and science teacher education programs to represent an important step in the advancement of PLTL pedagogical practice.

\section{$\underline{\text { In Conclusion }}$}

We have now completed two semesters of piloting the Virtual Peer-Led Mathematics Study Groups, and over three years of using Integrated PLTL. We believe that they represent viable modalities and potential alternatives to the traditional PLTL workshop. The study groups could be offered as a mandatory component of a given course, or student attendance could be voluntary. Common challenges on the virtual academic platform strongly suggest that institutions--especially those with nontraditional students--need to think about providing more flexible learning options and support structures for their students, and certainly the study groups offer one such example. Beyond that, alliances with mathematics and science teacher education programs and the recruiting of Peer Leaders from those programs could insure their extensive and continued pedagogical training. The integration of Peer Leader training in these programs, on the other hand, would provide these prospective teachers with an opportunity to select and sequence learning activities, and to enact small and whole-group discussions with diverse students--a benefit that we consider to be of inestimable value to teacher preparation programs everywhere.

\section{Acknowledgments}

We would like to acknowledge the pioneering work of our beloved late colleague Janet Liou-Mark, who was instrumental in the implementation of Peer-led Team Learning in our college, and whose dedicated work paved the way to employing and experimenting with various PLTL modalities. We would also like to thank the reviewers, whose graceful comments and thorough editorial work have helped improve the manuscript. 
References

Becvar, J. E., Dreyfuss, A. E., \& Dickson, W. E. (2008). Training faculty to train students in peer-led team learning. Workshop at 2008 38th Annual Frontiers in Education Conference, Saratoga Springs, NY.

Boaler, J., Humphreys, C. (2005). Connecting Mathematical Ideas. Middle School Video Cases to Support Teaching and Learning. Heinemann.

Brown, P., Sawyer, K. R., Frey, R. (2009a, April). Investigating Peer Leader discourse in peer-led team learning in general chemistry. Poster presented at the annual meeting of the American Educational Research Association, San Diego, CA.

Brown, P., Sawyer, K. R., Frey, R. (2009b, Oct). Peer-led team learning in general chemistry: Investigating the discourse of Peer Leaders and students. Paper presented at the annual meeting of the Mid-western Educational Research Association Conference, St. Louis, MO.

Chapin, S., O’Connor, C., \& Anderson, N. (2009). Classroom discussions: Using math talk to help students learn. Sausalito, CA: Math Solutions

Chase, A., Rao, A., Lakmala, P., Varma-Nelson, P. (2020). Beyond content knowledge: transferable skills connected to experience as a Peer Leader in a PLTL program and longterm impacts. International Journal for STEM Education, 7 (29), https: //doi.org/10.1186/s40594-020-00228-1

Clark, A., Raker, J. (2020). Peer Leaders' Perceived Roles: An Exploratory Study in a Postsecondary Organic Chemistry Course. International Journal of Teaching and Learning in Higher Education, 32 (2), 180-189.

Cracolice, M., \& Deming, J. (2005). Peer-led team learning: Promoting conceptual understanding and reasoning ability, published online for the Winter 2005 CONFCHEM: Trends and New Ideas in Chemical Education, retrieved from https: / / confchem.ccce.divched.org/2005WinterConfChem

Hill, H. C., Rowan, B., \& Ball, D. L. (2005). Effects of teachers' mathematical knowledge for teaching on student achievement. American Educational Research Journal, 42(2), 371406.

Hoiland, S., Reyes, S., Varelas, A. (2020). The impact of a Supplemental Instruction Program on diverse Peer Leaders at a two-year institution. Journal of Peer Learning, 13, 5-12. 
Hung, W., Jonassen, D. H., \& Liu, R. (2008). Problem-based learning. In J. M. Spector, M. D. Merrill, J. V. Merrienboer, \& M. P. Driscoll (Eds.), Handbook of Research on Educational Communications and Technology (3rd ed., pp. 485-506). New York, NY: Routledge.

Keup, J. (2016). Peer Leadership as an emerging high-impact practice: An exploratory study of the American experience. Journal of Student Affairs in Africa, 4(1), 33-52, DOI: 10.14426/jsaa.v4i1.143.

Kokkelenberg, E. C., Dillon, M., \& Christy, S. M. (2008). The effects of class size on student grades at a public university. Economics of Education Review, 27(2), 221-33, doi:10.1016/j.econedurev.2006.09.011

Lampert, M. \& Graziani, F. (2009). Instructional activities as a tool for teachers' and teacher educators' learning in and for ambitious practice. Elementary School Journal, 109, 491-509.

Lee, Y., \& Choi, J. (2010). A review of online course dropout research: Implications for practice and future research. Educational Technology Research and Development, 59(5), 593618. doi:10.1007/s11423-010-9177-y

Liou-Mark, J., Han, S., Ahmed, M., Anglade, F., \& Young, J. (2014). Strengthening foundational courses through the implementation of peer-led workshops. Conference Proceedings of the 2013 Peer- Led Team Learning International Society. Online: https: / / pltlis.org/wp-content/uploads/2015/10/Liou-Mark-Han-Ahmed-AngladeYoung-2013.pdf

Liou-Mark, J., Dreyfuss, A. E., Han, S., Yuen-Lau, L., \& Yu, K. (2015). AIM for success: Peer-led team learning supports first-year transition to college-level mathematics. Journal of Learning Development in Higher Education, (Special Issue: Peer Assisted Learning), 1-24. Plymouth, UK, 00. doi:10.47408/jldhe.v0i0.312

Liou-Mark, J., Ghosh-Dastidar, U., Samaroo, D., \& Villatoro, M. (2018). The peer-led team learning leadership program for first year minority science, engineering, and mathematics students. Journal of Peer Learning, 11 (1), 65-75. Retrieved from http: / / ro.uow.edu.au/ajpl/vol11/iss 1/5

Narode, R. (2012). Pair Problem-Solving. Peer-Led Team Learning: Leader Training. Online at http://www.pltlis.org. Originally published in Progressions: The Peer-Led Team Learning Project Newsletter, Volume 1, Number 3, Spring 2000.

Robertson, A., Scherr, R., Hammer, D. (2016). Responsive teaching in science and mathematics. New York: Routledge.

Schoenfeld, A. (2014)._What makes for powerful classrooms, and how can we support teachers in creating them? A story of research and practice productively intertwined. Educational Researcher. 43: 404-412. 
Snyder, J., Carter, B. E., \& Wiles, J. (2015). Implementation of the peer-led team-learning instructional model as a stopgap measure improves student achievement for students opting out of laboratory," CBE Life Sciences Education, 14(1), https: //doi.org/10.1187/cbe.13-08-0168.

Strobel, J., \& van Barneveld, A. (2009). When is PBL more effective? A metasynthesis of meta-analyses comparing PBL to conventional classrooms. Interdisciplinary Journal of Problem-Based Learning, 3(1). doi:10.7771/1541 - 5015.1046 